\title{
MINIMISATION OF MECHANICAL CROSS TALK IN PERIODIC PIEZOELECTRIC COMPOSITE ARRAYS
}

D. Robertson ${ }^{1}$, G. Hayward ${ }^{1}$, A. Gachagan ${ }^{1}$ and P. Reynolds ${ }^{2}$

${ }^{1}$ Centre for Ultrasonic Engineering, University of Strathclyde, Glasgow, UK;

${ }^{2}$ Weidlinger Associates Inc, Los Altos, U.S.A

\begin{abstract}
This paper describes an investigation into mechanical cross talk within 1-3 and 2-2 connectivity piezoelectric composite array configurations, comprising a matrix of active piezoelectric elements embedded within a passive, polymeric, material. One way to take full advantage of the reported sensitivity and bandwidth improvements from single crystal materials is to configure them as a piezoelectric composite. For this work, piezoelectric ceramic, lithium niobate and single crystal pmn-pt materials are investigated as the active component in the piezocomposite array designs. Within these piezoelectric configurations, the generation of ultrasonic inter-pillar modes, which arise due to the periodicity of the active piezoelectric elements within the piezocomposite lattice, can be detrimental to the array performance. Consequently, finite element (FE) modelling, using PZFlex, is utilised to provide design techniques for the removal of these inter-pillar modes from the frequency band of interest and the realisation of unimodal piezocomposite transducer structures. Further FE modelling is used to generate dispersion data for 2-2, and doubly periodic 1-3, composite substrates. This dispersion data is used to design the linear arrays, with the objective of minimising mechanical inter-element cross talk. A comparison between the FE predicted mechanical cross coupling between array elements, for each composite material operating in air, is supported by experimentally measured data. Subsequently, the validated FE models are extended to include both operation into a solid load and the introduction of a backing material to simulate the operation of a practical NDE array transducer. The design techniques obtained from PZFlex are shown to produce arrays with low cross talk and the extent of the cross talk in manufactured and modelled ceramic and pmn-pt single crystal arrays is compared.
\end{abstract}

Introduction: This paper investigates the design of 1-3 and 2-2 connectivity piezoelectric composite arrays. Piezoelectric composites comprise a matrix of active piezoelectric elements embedded within a passive, usually polymeric material, as shown in Figure 1.
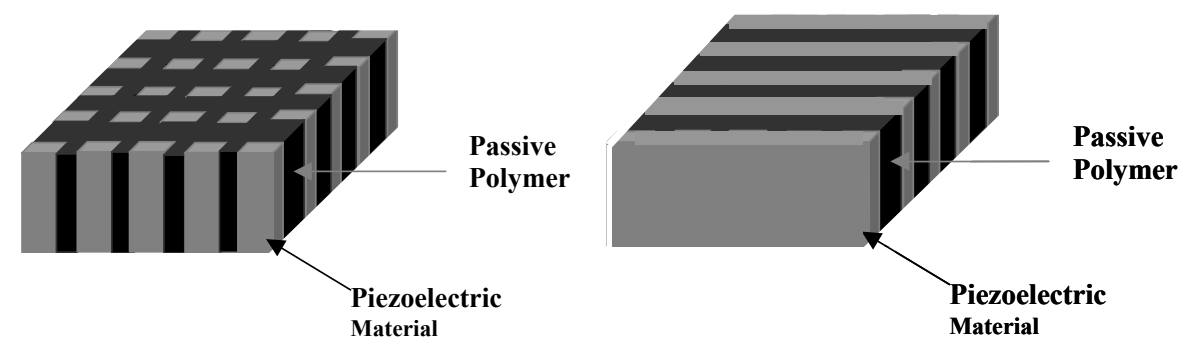

Figure 1 (a) and (b). Diagram of a 1-3 composite (left) and a 2-2 composite (right).

The work in this paper initially focuses on obtaining uniform surface displacements from a composite substrate and then focuses on obtaining low mechanical coupling between array elements patterned on to the substrate. To achieve these two separate design challenges two different types of waves have to be considered; inter-pillar waves and travelling Lamb waves.

Inter-pillar waves exist in any composite configuration due to the microstructure of the substrate. These waves are generated under the electroded areas and if these waves are coupled to 
the fundamental thickness operating mode as a consequence of their resonant frequencies being similar, the uniformity of the surface displacement can be severely degraded.

It is however travelling waves that are responsible for most of the coupling of energy between elements in a composite array. These travelling waves in solid materials are called Lamb waves. Lamb waves are perturbations propagating in a solid plate or layer, for which displacements occur both in the direction of wave propagation and perpendicular to the plane of the plate [1]. It is known that periodic 1-3 and 2-2 piezoelectric composite substrates support Lamb waves that propagate parallel to the plane of the substrate plate. The behaviour of Lamb waves in homogeneous plates of material is well known and understood, however the behaviour of Lamb waves in piezoelectric composite structures is less well documented.

In this work, arrays were made from 1-3 and 2-2 composite substrates. Firstly, finite element (FE) modelling, using the PZFlex code [2], was used to determine the frequencies of inter-pillar wave activity, which arise due to the periodicity of the active piezoelectric elements within the piezocomposite lattice. Design techniques to remove these inter-pillar waves from the frequency band of interest are used to produce unimodal piezocomposite transducer substrates. Next, the dispersion data for the composite substrates was taken into consideration to help design the arrays. These arrays were designed with the objective of minimising the mechanical cross coupling between the array elements. The mechanical cross coupling in the manufactured arrays was measured experimentally using a laser vibrometer and this data is compared to the mechanical cross coupling predicted using PZFlex. Further FE modelling was carried out to simulate the cross coupling of arrays operating in to a stainless steel load.

\section{Results:}

Inter-Pillar Modes: The phase velocities of the inter-pillar waves in 2-2 and 1-3 composites were modelled using PZFlex. The composite configurations modelled are summarised in Table 1. For each device the lateral dimensions are $5 \times 5 \mathrm{~mm}$. All piezoelectric composite devices described in this paper incorporate the low loss, hard setting epoxy CY1300/HY1301 as the passive filler material [3].

\begin{tabular}{|c|c|c|c|c|}
\hline Device & Configuration & Material & Kerf $(\mathrm{mm})$ & Thickness $(\mathrm{mm})$ \\
\hline A & $2-2$ & PZT5H & 0.045 & 0.35 \\
\hline B & $2-2$ & PZN8 & 0.068 & 0.37 \\
\hline C & $1-3$ & PZT5H & 0.045 & 0.4 \\
\hline D & $1-3$ & PZN4 & 0.093 & 0.4 \\
\hline
\end{tabular}

Table 1. Composite design parameters used in the FE modelling.

For each composite configuration, simulations were run at volume fractions between $10 \%$ and $90 \%$ in 10\% steps, resulting in 36 different simulations. The electrical impedance and surface displacement output spectrums were obtained from each PZFlex model. The frequencies of the inter-pillar modes were taken from the displacement output spectrum and converted to a phase velocity. Figure 2 shows the electrical impedance of device $C$, it can seen that the fundamental thickness mode at approximately $4.5 \mathrm{MHz}$ is unimodal. The electrical impedance of device $\mathrm{D}$ shown in Figure 3 also shows a unimodal thickness mode resonance at about $3.5 \mathrm{MHz}$ with no other modes coupled to the resonance. 


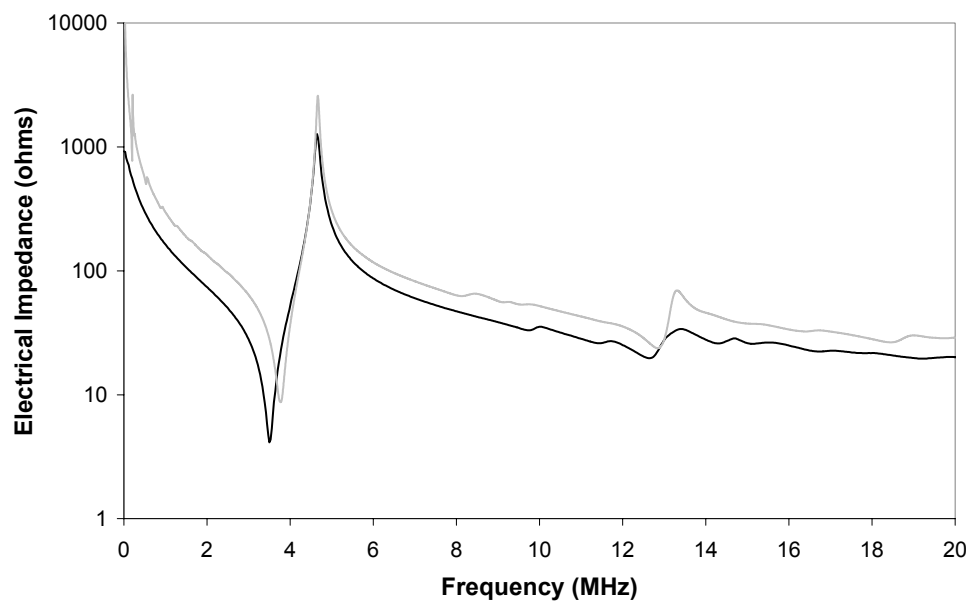

Figure 2. Modeled (black line) and measured (grey line) electrical impedance for the pzt5h composite, device $\mathrm{C}$.

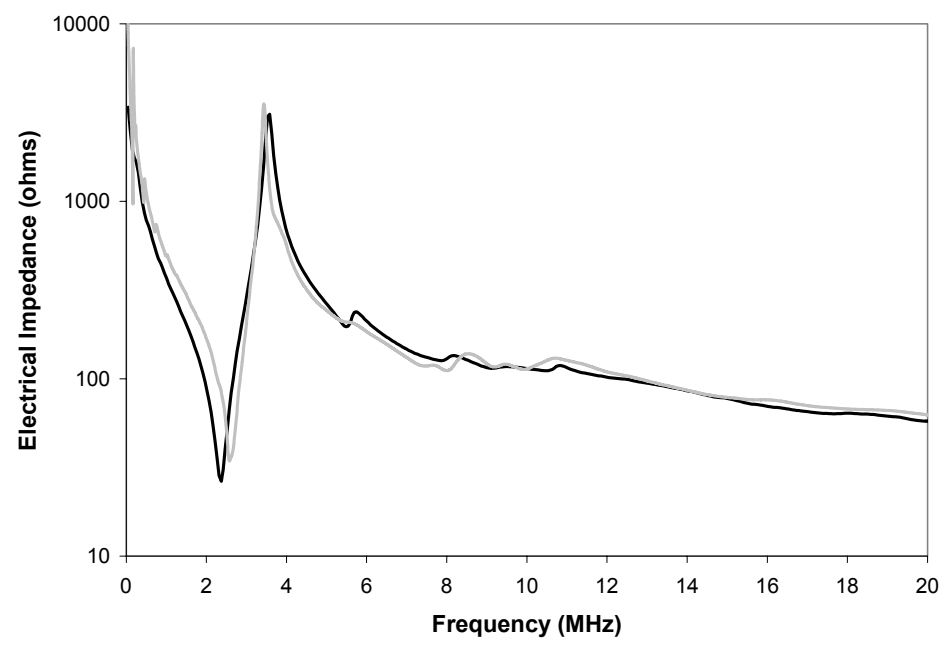

Figure 3. Modeled (black line) and measured (grey line) electrical impedance for the pzn-4.5\%pt composite, device D.

The inter-pillar mode for device $\mathrm{C}$ is evident in the experimental impedance data at approximately $8.1 \mathrm{MHz}$, well removed from the fundamental thickness mode. For device $\mathrm{D}$ the inter-pillar mode is evident at about $5.4 \mathrm{MHz}$, which is only $2 \mathrm{MHz}$ above the thickness mode but still well enough removed from the thickness mode not to affect the device behaviour under most drive conditions. The PZFlex modeling of the inter-pillar phase velocity highlighted differences between pzt5h and pzn-pt especially at higher volume fractions. Details on this work can be found in [4] and additional work in this area will be reported at a later date.

Piezocomposite Arrays: With the frequency of the inter-pillar modes in both single crystal and ceramic 1-3 and 2-2 composites more clearly understood it was possible to design substrates that should be unimodal over the desired frequency band. The next task was to investigate Lamb waves that could potentially couple mechanical energy between array elements that would be patterned on to a composite substrate. To determine the characteristics of the Lamb waves that 
could exist in the composite substrates under investigation, PZFlex was used to obtain Lamb wave dispersion curves.

Lamb Wave Dispersion Curves: To obtain the dispersion characteristics of all possible Lamb waves that could exist in the type of composites being investigated new PZFlex models were created. In these models composite plates were mechanically excited by a high frequency force delta function that was applied over 1 element of the finite element model. Hence the applied force function was both wideband in the frequency domain and in the wavenumber domain and could generate Lamb waves over all frequencies and wavenumbers of interest. The $\mathrm{x}$ and $\mathrm{y}$ displacements at each surface node were saved and this displacement data was used to obtain the dispersion curves for the composite plates. To obtain the dispersion curves shown in Figures 4 and 5 the surface displacement-time data saved from the PZFlex models was converted to the wavenumber-frequency domain via a 2D Fourier Transform [5].

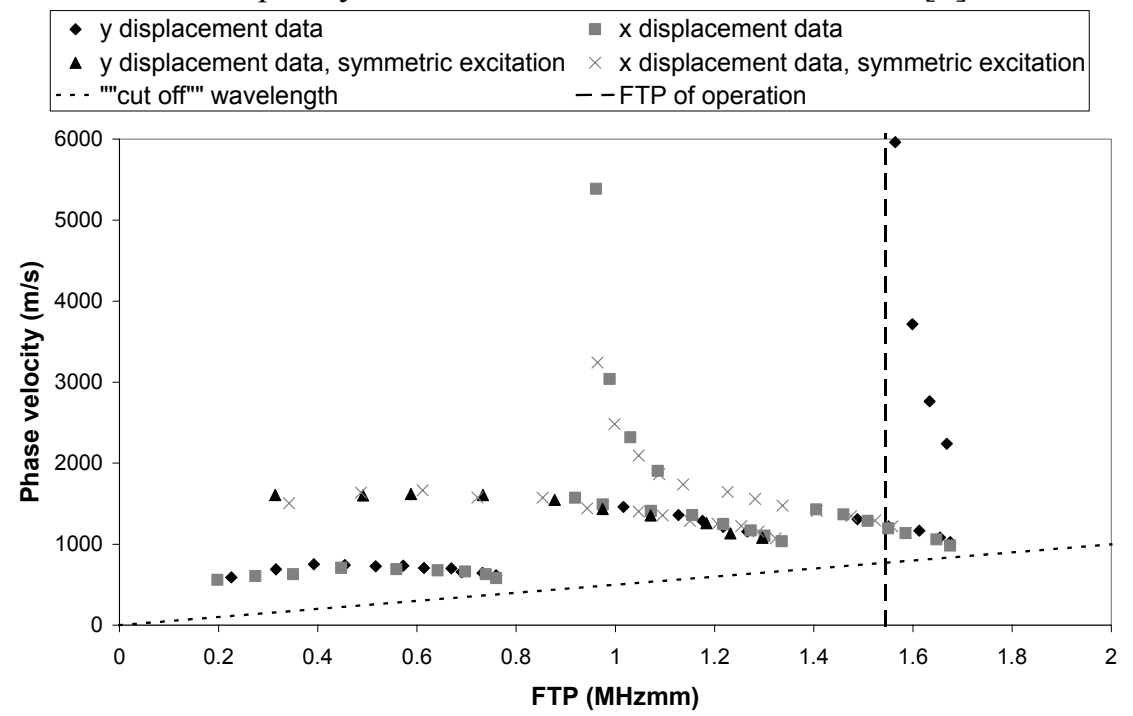

Figure 4. Lamb wave dispersion curve for a $50 \%$ volume fraction $2-2$ pzt 5 h composite.

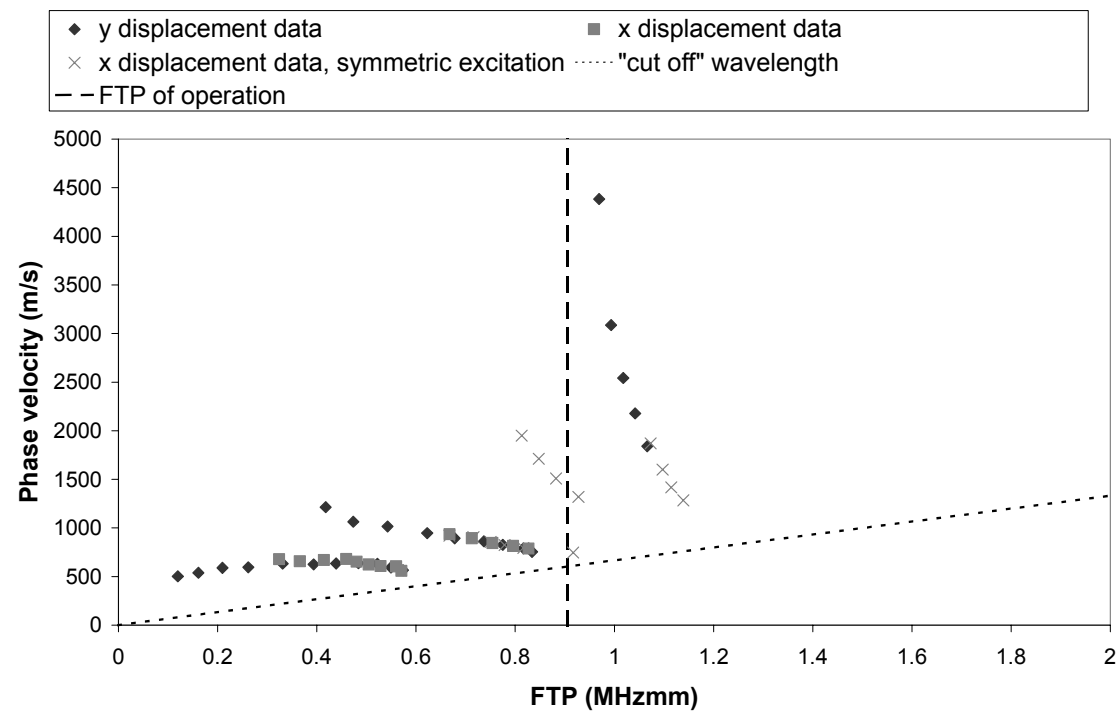

Figure 5. Lamb wave dispersion curve for a $50 \%$ volume fraction $2-2$ pzn- $8 \%$ pt composite. 
The dispersion curves in Figures 4 and 5 are both for 50\% volume fraction 2-2 composites. It can be seen that the same modes are present in the pzt5h based composite (Figure 4) and the pzn$8 \%$ pt based one (Figure 5). The modes shown are the $\mathrm{A}_{0}, \mathrm{~S}_{0}, \mathrm{~A}_{1}$ and $\mathrm{S}_{1}$ Lamb modes. The frequency of the Lamb modes as well as the fundamental thickness mode are considerably lower in the pzn- $8 \%$ pt composite than for the pzt5h composite due to the slower speed of sound in the single crystal material.

From analysis of these dispersion curves it was noted that all of the modes were "cut off" along a line of constant wavelength as indicated by the dotted line in Figures 4 and 5. No modes could be detected at any wavelength lower than the "cut off" line. This "cut off" wavelength is proportional to the pitch of the composite. Also, from the dispersion curves and subsequent PZFlex models of mechanical cross coupling in composite arrays it was determined that the $\mathrm{S}_{0}$ mode was responsible for the majority of the cross coupling. Work reported by Certon et al [6] also found that the majority of cross coupling in composite arrays was from the $\mathrm{S}_{0}$ mode.

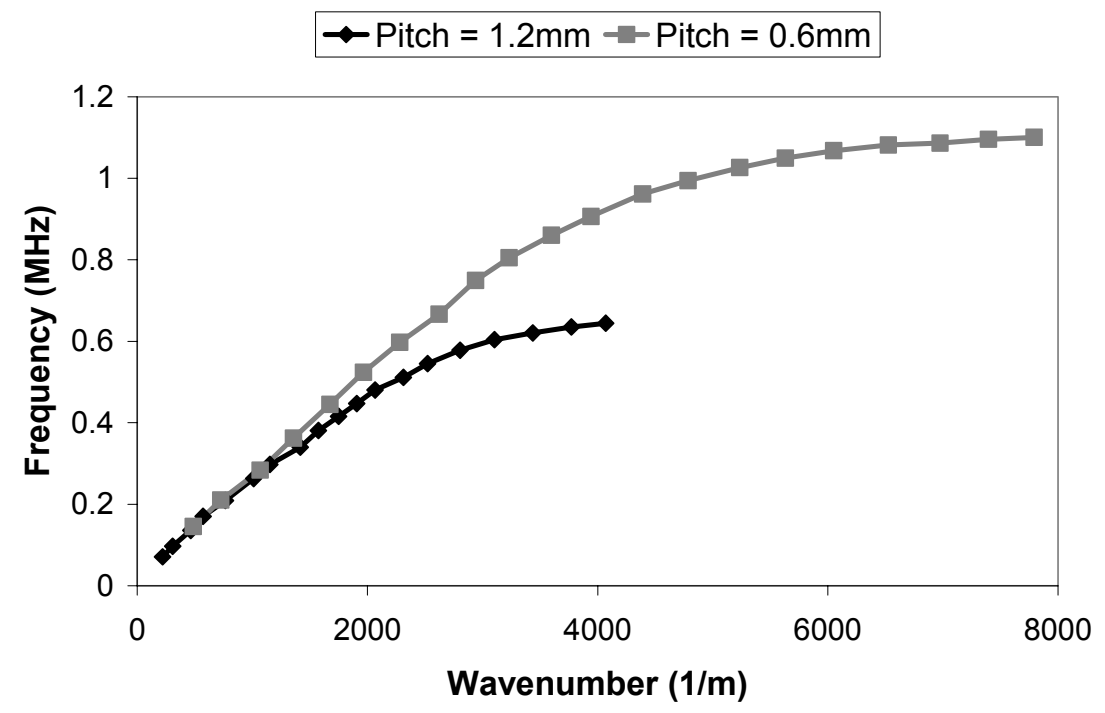

Figure 6. Simulated $S_{0}$ mode dispersion curves for two $50 \%$ volume fraction $2-2$ pzt5h composites.

Figure 6 shows $\mathrm{S}_{0}$ mode dispersion curves for two pzt5h composites. One composite has twice the pitch of the other and it can be clearly seen that this reduces the maximum propagating frequency by almost a factor of 2 . By designing composite arrays with a large pitch so that no Lamb waves of a frequency close to the array operating frequency can propagate the cross coupling between elements should be significantly lowered.

Cross coupling in Air: From the work described in the previous section it was stated that Lamb waves with a short wavelength compared to the composite pitch are "cut off" and cannot propagate. Therefore it was decided to test the effectiveness of this "cut off" by manufacturing both 1-3 and 2-2 composite arrays with a large pitch to inhibit the propagation of Lamb waves with a frequency close to the fundamental operating. Six arrays were manufactured but results are shown for only two devices, one based on pzt5h and the other on pmn-28\%pt. Table 2 shows the design parameters for these arrays, each array was $15 \times 15 \mathrm{~mm}$ square and had 5 elements. 


\begin{tabular}{|c|c|c|c|c|c|}
\hline Device & Configuration & Material & VF (\%) & $\begin{array}{c}\text { Kerf } \\
(\mathrm{mm})\end{array}$ & $\begin{array}{c}\text { Thickness } \\
(\mathrm{mm})\end{array}$ \\
\hline B & $1-3$ & PZT5H & 70 & 0.13 & 1.5 \\
\hline D & $1-3$ & PMN-PT & 70 & 0.13 & 1.5 \\
\hline
\end{tabular}

Table 2. Composite array design parameters.

To obtain the mechanical cross coupling for these devices the surface displacement was measured using a laser vibrometer with only the centre array element being electrically excited at the frequency of operation, which was $1.01 \mathrm{MHz}$ for both arrays.
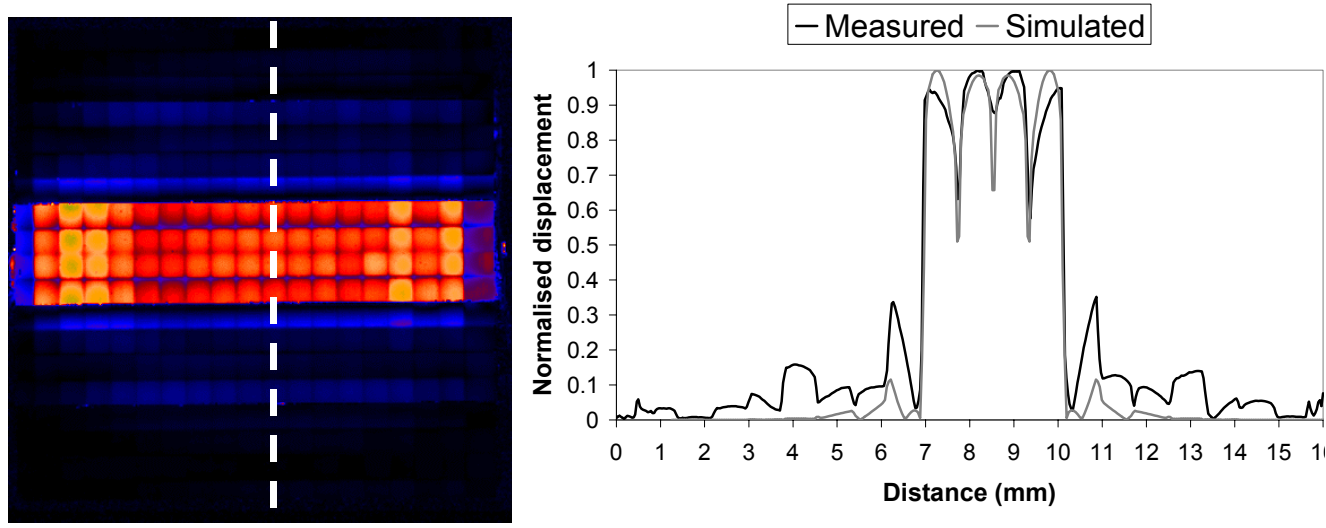

Figure 7 (a) and (b). Measured and Simulated Cross coupling for the pzt5h array.
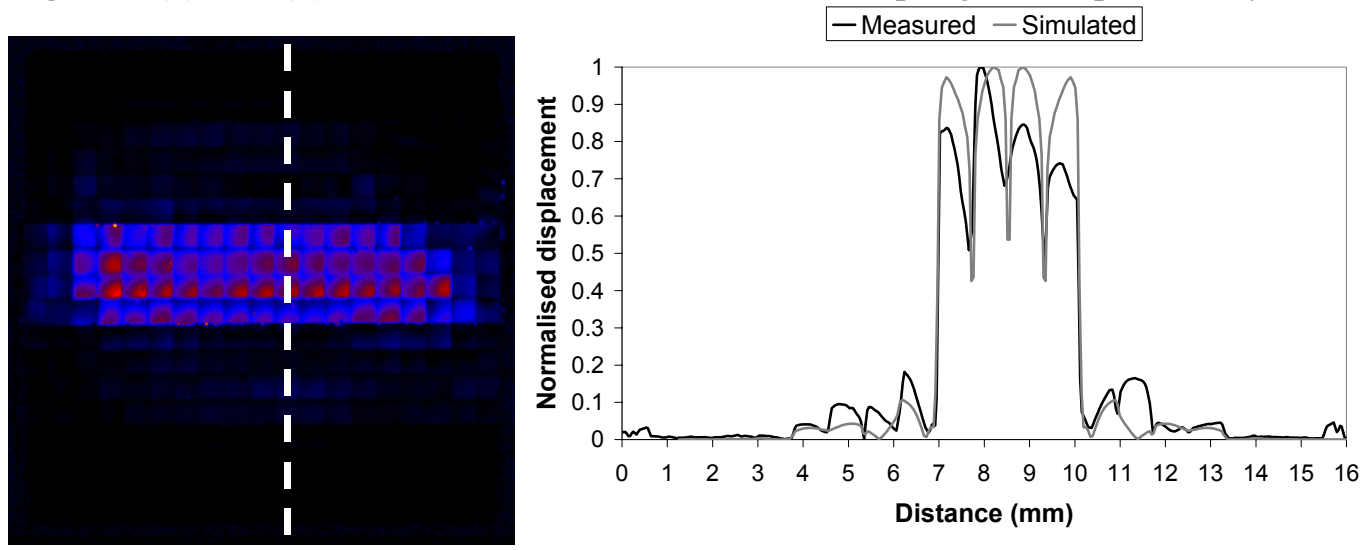

Figure 8 (a) and (b). Measured and Simulated Cross coupling for the pmn-28\%pt array.

Figure 7 (a) shows the measured surface displacement profile for the pzt5h based 1-3 array. The surface displacement cross section (Figure 7 (b)) shows both the measured displacement and the displacement predicted by PZFlex. The maximum coupling in to the adjacent array elements is $9 \mathrm{~dB}$ while the maximum cross coupling into the outer elements is much lower at $-23 \mathrm{~dB}$. The data presented in Figure 8 (a) and (b) is for the single crystal pmn-28\%pt array, the maximum measured cross coupling is $-15 \mathrm{~dB}$ in to the adjacent elements and $-27 \mathrm{~dB}$ in to the outer elements.

Cross Coupling into a Steel Load: To investigate how effective this technique would be for reducing cross coupling in a typical NDT situation, two further FE models were run with the devices operating in to a steel load and with a light backing layer. One of the simulated arrays was based on pzt5h while the other was based on the single crystal material pmn-pt. Both arrays had the same dimensions of $15 \times 15 \times 1.5 \mathrm{~mm}$ and had a kerf width of $0.18 \mathrm{~mm}$. However, the volume fraction for the pzt $5 \mathrm{~h}$ array was $77 \%$ while for the pmn-pt array it was $69 \%$. 


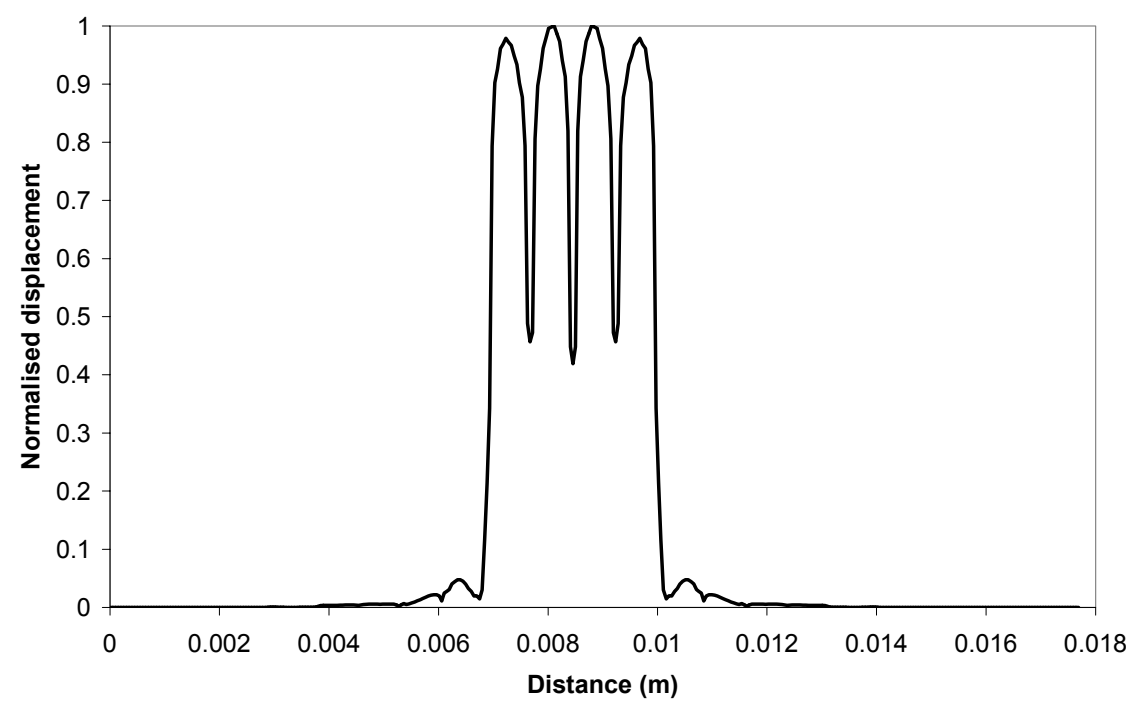

Figure 9. Simulated Cross coupling for a pzt5h array in to a steel load.

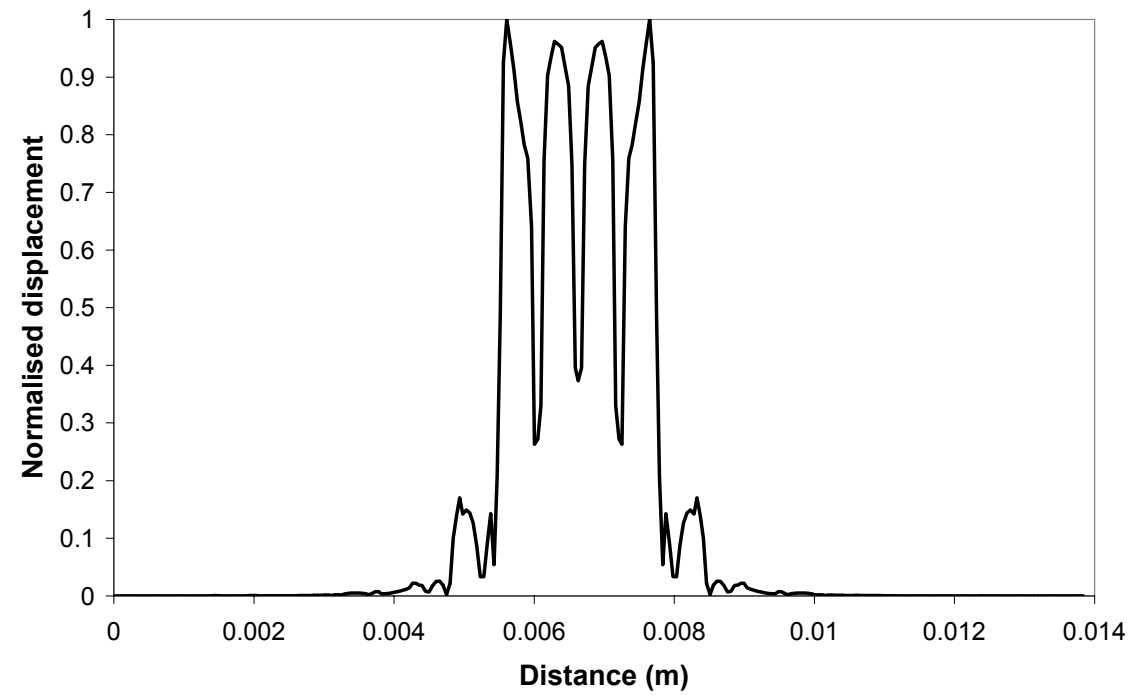

Figure 10. Simulated Cross coupling for a pmn-pt array in to a steel load.

Figure 9 shows the predicted cross coupling in the pzt5h array. The cross coupling is low with the maximum displacement in the adjacent elements being $-26 \mathrm{~dB}$ and $-63 \mathrm{~dB}$ in the outer elements. For the pmn-pt array the cross coupling is higher than for the pzt5h array with the maximum displacement in to the adjacent elements being $-15 \mathrm{~dB}$ and $-46 \mathrm{~dB}$ in the outer elements.

Conclusions: This paper investigated two types of waves that can exist is piezocomposite arrays. The first kind of waves to be investigated were inter-pillar waves in the microstructure of the composite substrate. PZFlex finite element simulations were developed to predict the inter-pillar phase velocity of these waves and have highlighted differences in the phase velocities for pzt5h and single crystal pzn-pt composites. This information on the inter-pillar phase velocities was used to help design composite substrates that were unimodal at the frequency of operation.

The second group of waves to be investigated were travelling Lamb waves that are responsible for much of the cross coupling in arrays. This paper investigated the minimisation of 
mechanical cross coupling in piezoelectric composite array transducers by designing the composite substrates to have a large pitch. This was successful in that the cross coupling encountered in each array was low for each array that was manufactured. The cross talk was found to be comparable in the pmn-pt and pzt5h arrays. This work has illustrated design techniques for producing piezoelectric array transducers from single crystal materials. The work will now be applied to the design and manufacture of practical transducer arrays in the field of NDT.

\section{References:}

[1] I. A. Viktorov, "Rayleigh and Lamb waves - physical theory and applications", Plenum Press, New York, 1967, chapter 2.

[2] PZFlex: Distributed by Weidlinger Associates, 4410 El Camino Real, Suite 110, Los Altos, CA, 94022, U.S.A.

[3] http://www.cue.ac.uk

[4] D. Robertson, G. Hayward and A. Gachagan, "Lamb Wave Suppression in Periodic 1-3 Piezoelectric Composite Transducers", 2002 Ultrasonics Symposium Proceedings, Pages 11521155.

[5] D. N. Alleyne and P. Cawley, "A two dimensional Fourier transform method for measurement of propagating multi-mode signals", J Acoust Soc of Amer 89(3), 1991, pp 1159-1168.

[6] D. Certon et al, "Investigation of Cross-Coupling in 1-3 Piezocomposite Arrays", IEEE Trans.

Ultrason., Ferroelect., Freq. Contr., vol. 48, No. 1, pp. 85-92, 2001. 